\title{
Therapeutic Endoscopic Procedures on a Human Cadaver-A Pilot Feasibility Study
}

\author{
Avinash Bhat Balekuduru ${ }^{1}$ Ashwini C. Appaji ${ }^{2}$ \\ 1Department of Gastroenterology, M. S. Ramaiah Hospitals, \\ Bangalore, Karnataka, India \\ ${ }^{2}$ Department of Anatomy, M. S. Ramaiah Hospitals, Bangalore, \\ Karnataka, India
}

\begin{abstract}
Address for correspondence Avinash Bhat Balekuduru, MD, DNB, DM, Department of Gastroenterology, M. S. Ramaiah Hospitals, Bangalore-560054, Karnataka, India (e-mail: avinashbalekuduru@gmail.com).
\end{abstract}

J Digest Endosc 2021;12:36-42.

\begin{abstract}
Keywords

- cautery

- clipping

- endoscopic training

- injection

- PEG

- polypectomy

Introduction The traditional apprentice model of teaching therapeutic endoscopic skills is inadequate due to unpredictable frequency and high stakes for patient outcome. Simulation had gained widespread acceptance for training modules. But all the procedures cannot be trained on simulator. We designed a novel human cadaver hands-on training module for practicing percutaneous endoscopic gastrostomy (PEG), endoscopic variceal band ligation (EVL), endoscopic injection (EI), snare polypectomy (SP), thermal cautery (TC), and endoscopic clip (EC) placement.

Methods A single preserved pre-coronavirus disease 2019 human cadaver was used as a training model. Twelve trainees ( 6 teams) used the module to acquire and practice new skills of PEG, EVL, EI, SP, and EC using standard endoscope and regular endoscopic accessories. All the trainees completed the course evaluation using a 5-point Likert scale $(5=$ strongly agree).

Results The training resulted in a self-reported increase in equipment familiarity and all the trainees felt uniformly that they are better prepared for performing the procedures on real patients. They strongly agreed that this exhaustive hands-on exercise has more educational value than attending lectures.

Conclusion Human cadaver can be used for trainees to hone therapeutic endoscopic skills by teaching modules with predefined learning objectives.
\end{abstract}

\section{Introduction}

The training of endoscopic skills on live patients in endoscopic unit under supervision of attending physician can cause potential harm to the patient. ${ }^{1}$ The direct observational tools can individualize the learning curve of each trainee to develop competence in particular endoscopic skill. ${ }^{2}$ Repetitive practice at their own learning pace is a major advantage of a simulator. It also provides a risk-free environment as compared with stressful real patient scenario., ${ }^{2,3}$ Simulation training provides experience in endoscopic skills to manage in real-life scenarios. ${ }^{4}$ The four major types of simulators are mechanical models (made of nontissue-plastic/wire materials that lack haptic feedback), live animal models (swine commonly used, but labor intensive), composite animal organ simulators using explanted organs (cannot mimic real-life scenarios), and virtual reality trainers (expensive) that can limit their use. ${ }^{2}$ But the use of human cadaver for endoscopic training is not well studied.

The role of the teacher is to break down the steps of the procedure with verbal communication and repetitive instruction to let the trainee master the procedure eventually. ${ }^{2}$ published online

February 21, 2021
DOI https://doi.org/

$10.1055 / \mathrm{s}-0041-1728223$ ISSN 0976-5042
(C)2021. Society of Gastrointestinal Endoscopy of India.

This is an open access article published by Thieme under the terms of the Creative Commons Attribution-NonDerivative-NonCommercial-License, permitting copying and reproduction so long as the original work is given appropriate credit. Contents may not be used for commercial purposes, or adapted, remixed, transformed or built upon. (https://creativecommons.org/licenses/by-nc-nd/4.0/).

Thieme Medical and Scientific Publishers Pvt. Ltd. A-12, 2nd Floor, Sector 2, Noida-201301 UP, India 
The simulator by itself has the feedback to allow repeated practice by trainees without the continuous presence of a trainer. ${ }^{5}$ But predefined learning goals have to be defined and trainer feedback is important in endoscopic training. ${ }^{6,7}$ Repetition and feedback as effective learning methods can improve cognitive/technical skills, lessen the learning curves, with faster skill acquisition especially in a human cadaver laboratory. It also offers a lifelike platform to learn through hands-on practice rather than standard bed side learning. The frequency and exposure of the procedures between one training institution to another may be different.

The aim of the study was to assess the feasibility of human cadaver in enhancing endoscopic skill in percutaneous endoscopic gastrostomy (PEG), injection, banding, polypectomy, cautery setting, and clipping.

\section{Materials and Methods}

\section{Cadaver Environment and Endoscope}

All human cadaveric endoscopic procedures in this feasibility pilot study were performed at M.S. Ramaiah Advanced learning center (MSR ALC), Bangalore. The fundamental steps of endoscopic skills- scope handling, checking scope- knobs / air/water/ suction function before intubation, maintaining luminal view, use of distension/ suction/ lens washing, retroflexion and passage of scope in to second part of duodenum were trained in supine position of cadaver. ${ }^{1}$

\section{Biosafety Level}

The human cadaver used in the study module was negative for blood-borne virus test (human immunodeficiency virus, hepatitis $B$ and $C$ ) and was stored before the onset of the coronavirus disease 2019 (COVID-19) times. All the delegates had personal protective equipment (laboratory gown, two pairs of gloves, face and eye protection, and a N-95 mask covering mouth and nose completely) along with social distancing norms.

\section{Training on Human Cadaver}

A forward viewing single channel gastroscope (GIF 150, Olympus, Tokyo, Japan) with an inbuilt light source processor (Olympus; CV-170 LED/HD) was used for the training module.

The earlier attempts to intubate a supine cadaver were difficult at the postcricoid area. A modified entry in to the esophagus was made from the left by a neck incision (-Fig. 1)

\section{Training Module Curriculum}

The training module structure is given in - Fig. 2. The training module started with an hour of interactive didactic lecture along with discussion on the preprocedure (indications, contraindications), intraprocedure (procedural steps), and postprocedure (early and delayed complications). Later the model was used as a workshop ( 4 hours) with six teams (2 trainees
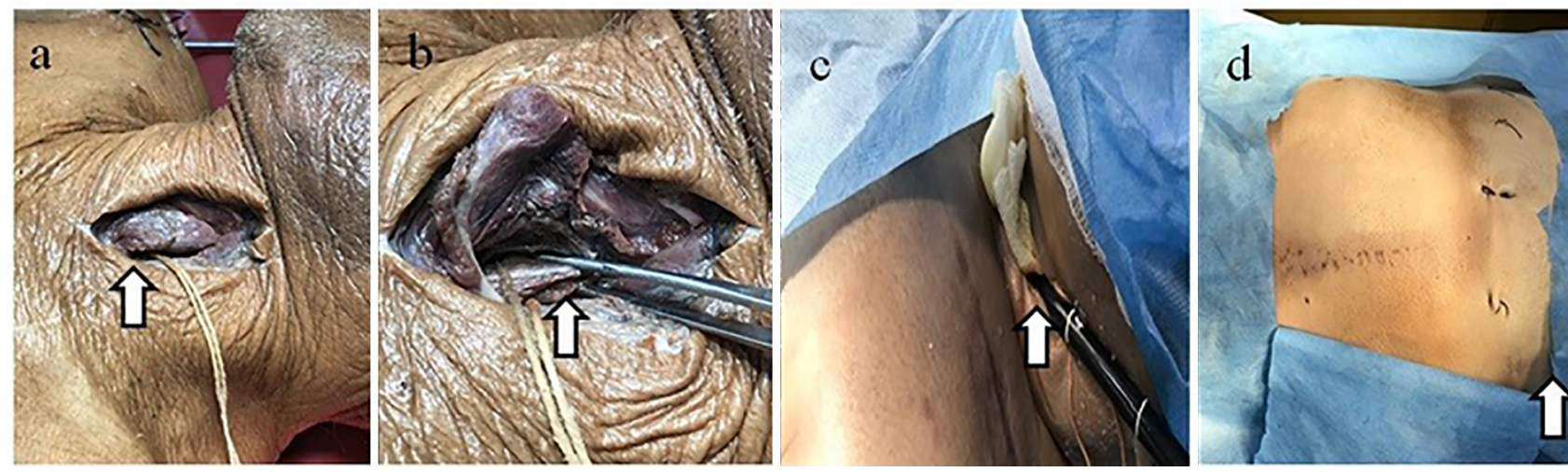

Fig. 1 (a) Modified neck incision showing thyroid cartilage and thread delineating the esophagus. (b) The esophageal lumen delineated by forceps and thread after exposure of thyroid cartilage. (c) Endoscope passed through the neck incision. (d) As earlier feedback was related to cadaver environment, only abdomen and neck of the cadaver were exposed with the remaining body that was draped (bold white arrow).

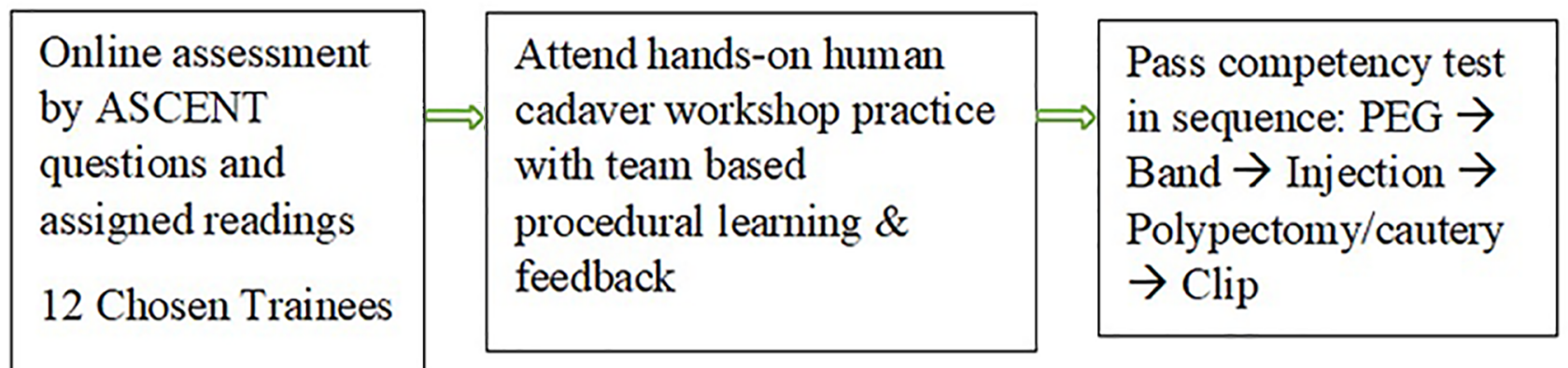

Fig. 2 Training curriculum flowchart. PEG, percutaneous endoscopic gastrostomy. 
each) and a mentor followed by an hour of debriefing session. The teams were divided as performing, assisting, and commentary teams. The remaining three supporting teams are watching the key steps of the procedure and correcting whenever necessary. The training approach was based on directly observed procedural skill training ${ }^{8}$ and Pendeltons rules. ${ }^{9}$ Trainees underwent supervised hands-on endoscopic session, to acquire the pre-designated endoscopic skill through focused repetition and feedback. ${ }^{10}$

The training module had five advanced endoscopic techniques: PEG placement, endoscopic injection (EI), endoscopic band ligation (EVL), snare polypectomy (SP) of simulated polyps, thermal cautery (TC), and endoscopic clipping (EC) of the mucosal defects. All the procedures were performed on a single human cadaver by 6 teams.

\section{Percutaneous Endoscopic Gastrostomy Skill}

The procedure steps were by two-person Ponsky Gauderer pull technique using $24 \mathrm{Fr}$ gastrostomy tube (Wilson-Cook, Winston-Salem, North Carolina, United States) and are described in - Figs. 3 and $\mathbf{4}$. $^{11}$

EVL skill: Multi-Band Ligator (Wilson-Cook; 6 shooter) was used for the EVL skill development. The barrel has to be fixed to the tip of the scope properly. Wall suction with white/red out (significant tissue is suctioned into the barrel) and release of band on the gastric folds (considered as varices) were performed. One band has to be released by clockwise rotation of the handle. The applied bands were considered as simulation gastric polyps are described in -Fig. $\mathbf{5}$.

EI skill: The injector used was injector force max (NM-400L-0423; $4 \mathrm{~mm} 23 \mathrm{G}$ needle). The skills required to perform include checking the needle-in/out and patency with normal saline of $2 \mathrm{~mL}$ (dead space volume), injector has to be passed down the working channel to the target site, communication with right hand assistant for the deployment of needle and the critical step of injection to the desired plane. Four-quadrant injection was chosen around the simulated polyp with submucosal elevation as described in - Fig. 6.

SP skill: Proper application of the snare (snare master SD-210 U-25; Olympus) through the working channel below the band of the simulated polyp along with good communication with the assistant. The snare should be able to tent the lesion and should not be overtight nor to be loose as in -Fig. 7.

TC skill: Cautery machine (Olympus ESG-300 with APU upgradation compatibility) was used for the polypectomy. The skill required was to connect to the snare with electrocautery and to deliver proper thermal energy for polypectomy. The cautery settings used were 40 pulse cut fast and forced coagulation with effect 2 . The endoscope has to be stabilized at proper angle and force, with probe tip closer to scope tip to apply firm and steady pressure on snare for polypectomy.

EC skill: The clip used for training was HX-110LR with applicator HX-610-090L (Olympus). The skill included proper loading of clip on to the introducer, which has to be then passed into the working channel. Proper clip application to the post polypectomy mucosal defect was done by changing the angle of endoscope. The target was positioned perpendicular and suction was used for definite clip application as in - Fig. 8.

An anonymous course evaluation survey using a 5-point Likert scale ( 1 = strongly disagree, 5 = strongly agree) was filled by the participating trainees. The study was approved by institute ethical board.

\section{Results}

Twelve trainees (11 final-year postgraduate students and one fourth year fellow of medical gastroenterology) received training in this model. All the trainees uniformly agreed that using real accessories and normal human anatomy for learning endoscopic skills has more educational value. The educational content delivery was better than traditional lectures
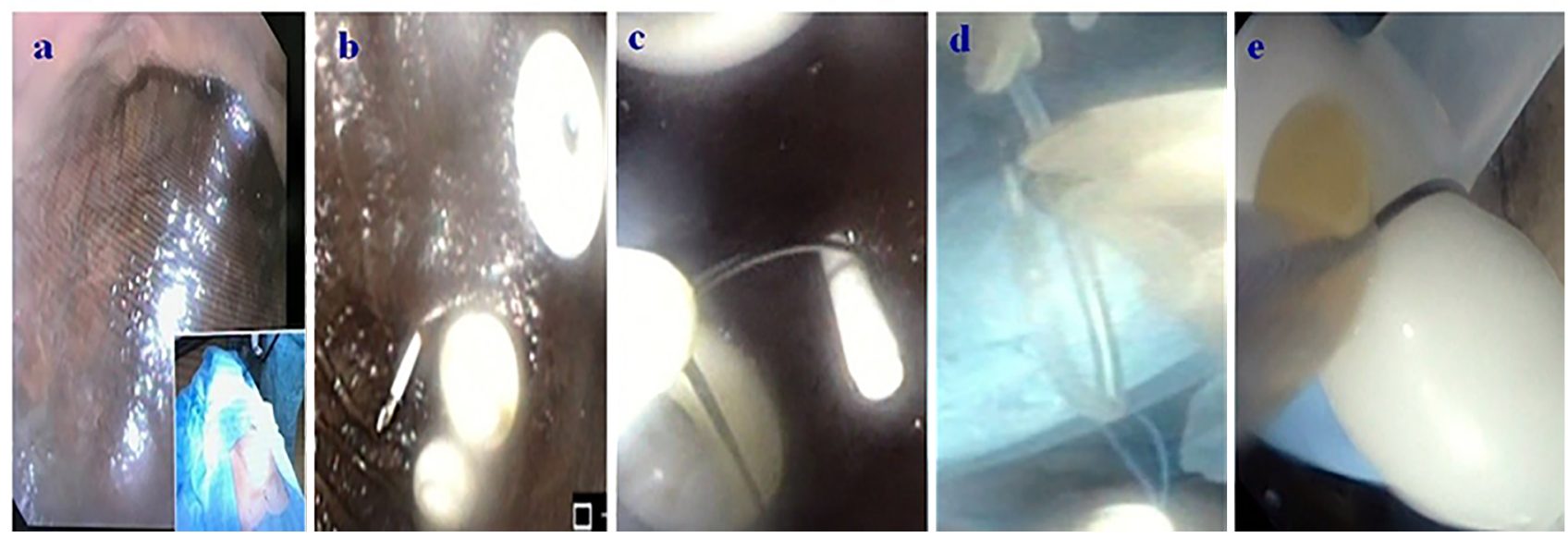

Fig. 3 (a) Maximal gastric distension and transillumination on anterior stomach wall (critical step) and digital 1:1 pressure (percutaneous endoscopic gastrostomy [PEG] site identification). (b) Puncture was made with an angiocath (22G x 1 1/2") after skin incision (fourth team PEG site). (c) A ready opened cold snare at the anticipated entry site on endoscopy side was used to catch the catheter. (d) A figure of 8 knot attaches the guidewire and PEG tube wire. (E) Snare was reused to tightly hold the internal bumper of the PEG tube that aids in easy intubation of the scope. 

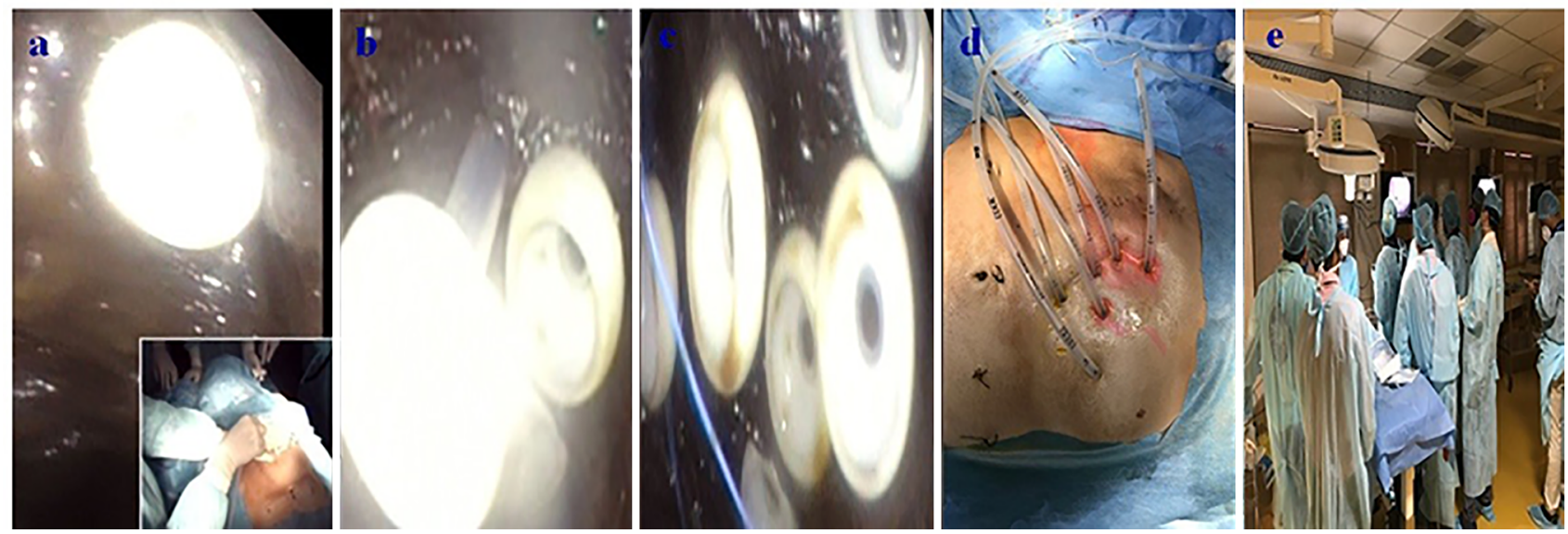

Fig. 4 (a) The inner bumper was fixated at $5 \mathrm{~mm}$ from gastric wall with appropriate pull-on abdominal end. (b) Troubleshoot/wrongly placed third percutaneous endoscopic gastrostomy (PEG) inner bumper. (c) Endoscopic picture of all six bumpers. (d) Abdominal end showing all six PEG tubes. (e) Six teams at operating room.
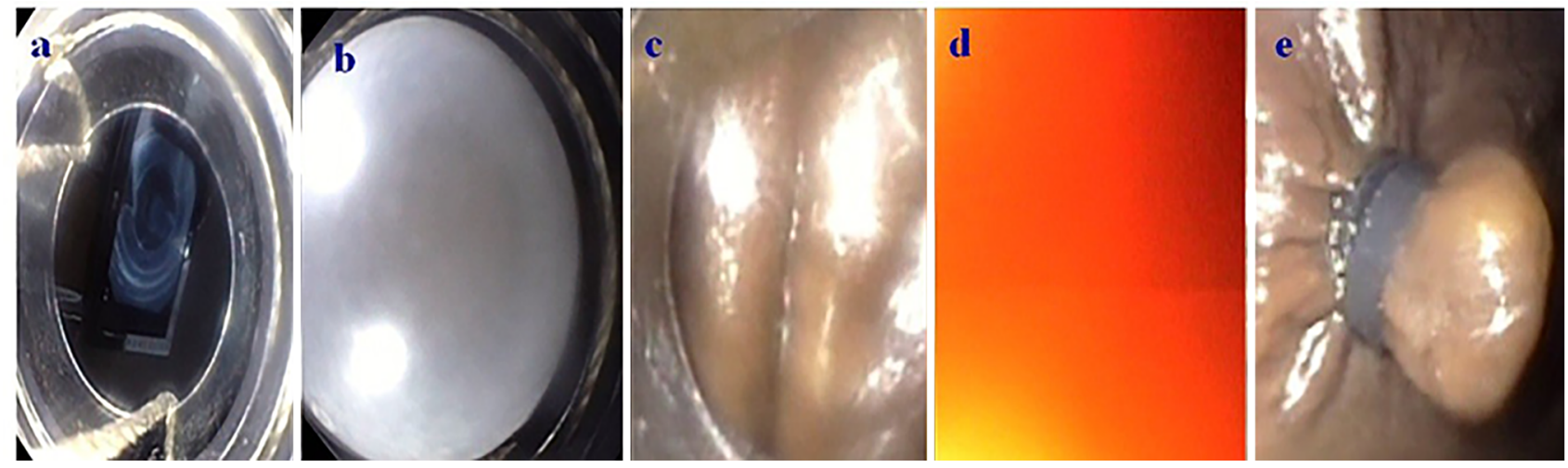

Fig. 5 (a) Proper fixation of banding cylinder at the end of the scope. (b) White out of the glove to secure proper suction before intubation. (c) Considering gastric folds as varices. (d) Red out of the mucosa to ensure significant tissue is suctioned before band deployment. (E) Troubleshoot of two bands deployed by double rotation that simulates a polyp.
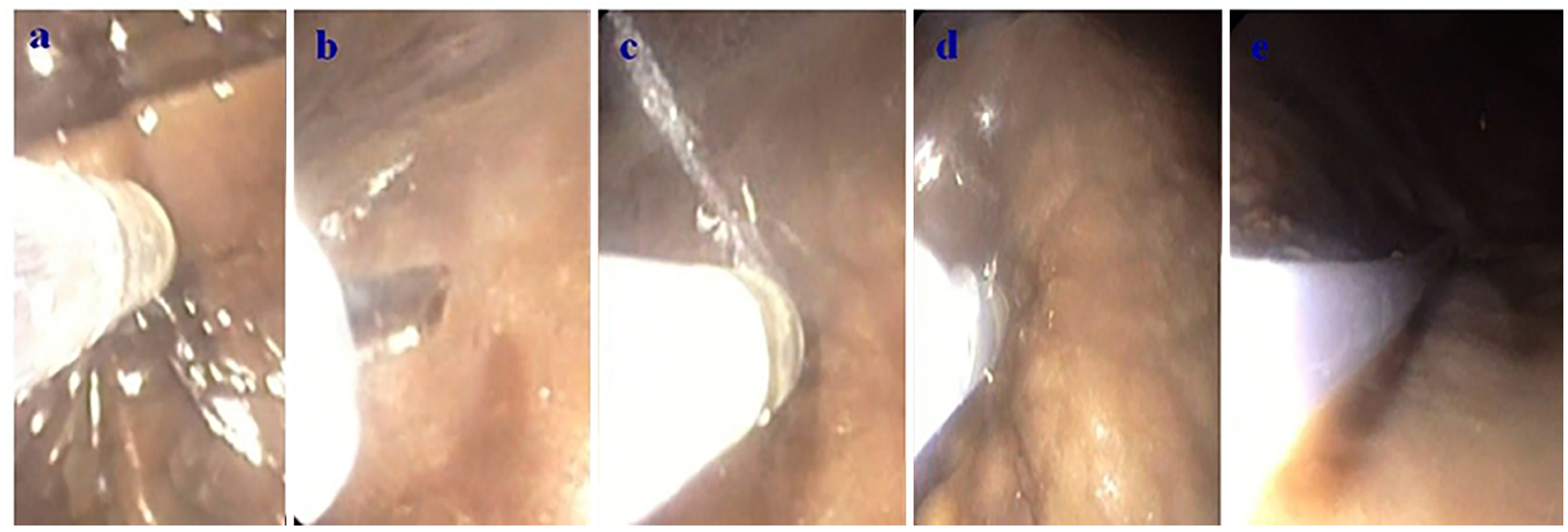

Fig. 6 (a) Proper positioning of the needle at desired site. (b) Correct command of needle out/in after sheath out. (c) If submucosal plane is not achieved, puncturing while injecting was followed to get the proper plane as in parts (d, e). Troubleshoot of wrong injection method far away from scope without control.

and reading alone. All have agreed that they had exhaustive hands-on time in the workshop and never had such an experience. Three $(25 \%)$ of them had first time performed EC, SP, TC procedures. Eleven of the 12 trainees agreed to come for second time for another module. The remaining trainee was concerned of the cadaver environment and COVID-19 times. All the trainees felt that the procedure skills were similar to real-life environment. All of them agreed that they are better 

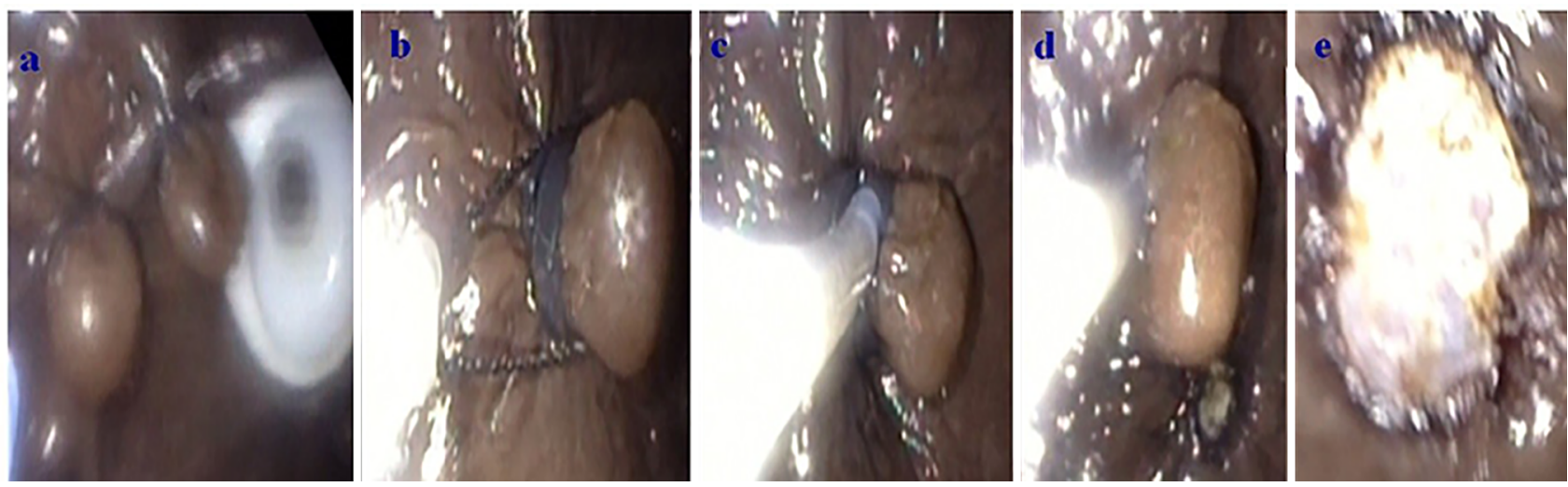

Fig. 7 (a) Simulated polyps adjacent to peg tube bumper. (b) Correct positioning of electrocautery snare at the base of polyp. (c) Tenting the polyp away from the base. (d) Resection of polyp and removal of polyp. (e) Postpolypectomy base to watch for target sign.
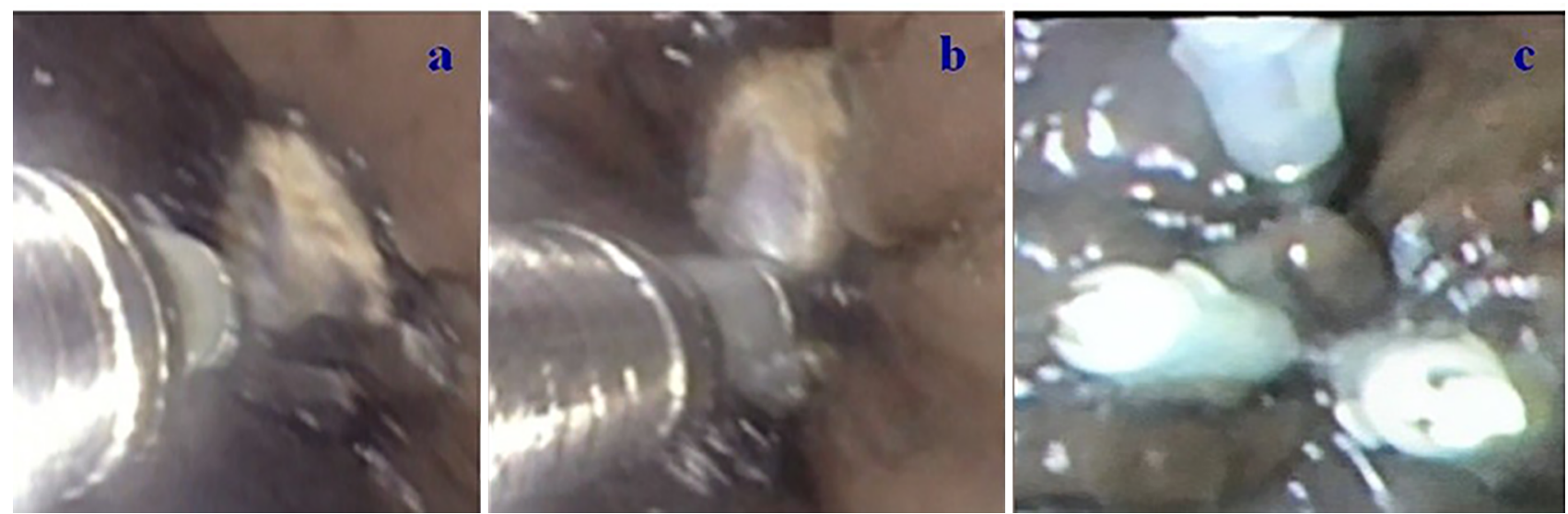

Fig. 8 (a) Proper endoscopic position and clip opening perpendicular to the mucosal defect. (b) Opposing the edges of the defect with the clip. (c) Multiple clips used to close the polypectomy site.

Table 1 Potential advantages of using human cadaver in training advanced endoscopic skills

\begin{tabular}{|c|c|c|}
\hline Sl. no. & Skill set & Potential advantages on the human cadaver \\
\hline 1 & PEG placement & $\begin{array}{l}\text { Real experience; multiple trainees were involved at the same time, training as an assis- } \\
\text { tant to endoscopist; instant performance feedback by mentor }\end{array}$ \\
\hline 2 & Band ligation & $\begin{array}{l}\text { Facilitate cooperation between trainees; exposes unforeseen band application-double } \\
\text { band placement/slippage of bands }\end{array}$ \\
\hline 3 & Injection & $\begin{array}{l}\text { Allows trainee to get the haptic feel of the amount of pressure required to get the } \\
\text { desired submucosal plane and how to correct it }\end{array}$ \\
\hline 4 & Snare polypectomy & Quick feedback, less risks, allows snaring of polyp at difficult and every possible location \\
\hline 5 & Electrocautery & Actual patient is safe; programmed cautery settings can be presented \\
\hline 6 & Endoscopic clipping & $\begin{array}{l}\text { Proper clip application to mucosal defect; reproducible; aids in decision making; train- } \\
\text { ees only loaded and applied the clip }\end{array}$ \\
\hline
\end{tabular}

Abbreviation: PEG, percutaneous endoscopic gastrostomy.

prepared for performing the procedures on real-life patient setting after having undergone the structured simulation program.

PEG placement: All the trainees felt that for choosing PEG site selection, transillumination was not feasible in spite of maximal gastric distension but due to thick abdominal wall of the chosen cadaver. PEG site was chosen by finger indentation and guiding needle techniques. The embalming solution was greasy that led to masking of the scope vision, requiring cleaning of the lens very often. The rest of the procedure steps were similar to procedure on real patients.

EVL, SP, TC, and EC procedures: The procedures had the real-life feel of the trigger for band release, positioning of snare for electrocautery, and clip application for the mucosal defect. The only differences were that it was easier to cut and clip was not opposing significant tissue as compared with live patients. 
Injection: In 48 injections, 10 (20\%) did not have the submucosal lift in the practice of 4 quadrant injection. The possible reason was wrong plane of injection due to application of more pressure on the needle. Once the assistant is injecting the saline, pricking at the same time, they were able to get the submucosal plane.

Complications: All the trainees were able to cut the simulated polyp below the band and none of them had a full thickness perforation. Bleeding could not be simulated in the current module. All of them felt that they were more confident in cadaver environment as there would be no complications. They also felt that coordinated skill development is easier in Pendleton model as they can discuss with their colleagues and remembered their schooling days.

\section{Discussion}

Endoscopic skill development in trainees is based on apprenticeship model on patients rather than on simulation model. Majority of the programs lack structured training modules for repetitive learning of interventions. Though endoscopic simulators can be used for training, they are not available in many teaching hospitals. In this study, the aim was to improve PEG, EI, SP, TC, EVL, and EC skill module involving 12 trainees with mentor feedback on a human cadaver.

The use of human cadaver in training endoscopic skills can help trainees to understand therapeutic application of accessories. The endoscopist should understand all the accessories better than anyone else in the endoscopic room. The ability to effectively communicate with endoscopic nurses/technicians can also be trained in human cadaver environment.

The GI mentor II (Simbionix USA. Inc., Cleveland, Ohio, United States) includes training modules on polypectomy, clipping, and gastrointestinal bleeding. Live porcine and canine models mitigate usage for training due to cost and ethical concerns. The Erlangen Active Training Simulator for Interventional Endoscopy and Grund model have either prepared porcine or artificial tissue models with perfusion pump to simulate bleeding. But there is no reported scientific data about use of human cadaver model in endoscopic skill development. ${ }^{12}$

Virtual reality simulators can simulate SP and EC skills, but the assessment and feel of the tissue are only possible in human tissue. However, there are no simulation models for PEG placement. Human cadaver model can fill in the lack of PEG training model as simulators lack the haptics of normal tissue.

In terms of feel of the tissue, trainees felt that learning in simulated environment was helpful for EVL, EI, EC, and SP. The only observation was that the polypectomy cut was faster than live human tissue. Another important factor in training was task variability that is common in live endoscopic procedures. The presence of mentor with changing modules for each team increased the exposure for troubleshoot/complications at each critical step that can enhance skill acquisition.

Earlier studies showed significantly higher procedural success rate and a nonsignificant reduction in occurrence of complications in clinical practice on simulator training. ${ }^{13}$
Multiple single-day intensive hands-on training in endoscopic therapeutic courses leads to significant improvement in trainee skills and is beneficial to integrate simulation models to actual supervised endoscopic skill training. But the minimum number of procedures needed to acquire and maintain the skill set is not known. ${ }^{4,5,14,15}$

Supplementation of teaching with prerecorded videos as part of ASGE learning center (http://www.asge.org) and online in DAVE project (Digital Atlas of Video Education, http://www.daveproject.org) can also be done.

The study has several strengths. It is the first innovative human cadaver endoscopic training model without any prior reported literature. Single model was used for training PEG, EVL, SP, EI, TC, and EC skill development, feedback, and increased fellow's basic knowledge. The use of the regular accessories, improvement in endoscopic skills, and feel of normal tissue were uniformly accepted by all the trainees. The module had low frequency high-risk procedures and trainees were better prepared for emergency endoscopic interventions.

The study has several limitations. It is a single-center study with limited study group of 12 trainees. The study was not powered to detect the difference in DOPS scores before and after the training module. All the trainees did not have uniform procedural skills. The absence of esophageal intubation, the supine positioning of the cadaver, and the endoscope had to be cleaned repeatedly for better vision as the preservative was greasy. Bleeding as a complication could not be emulated. Though after the experiential learning of the skills and receipt of the feedback in the simulated environment, how it will translate to real-life environment was not studied.

Further work needs to be done to improve the utility of this model within the teaching curriculum. The endoscopic visibility has to be improved probably by precleaning the stomach by a detergent. There should be better access for esophageal intubation. There is scope of development of other training modules like technique of endoloop, placement of different foreign bodies (FB) in stomach and train in FB retrieval methods; creation of neo-pylorus to simulate stricture for dilation. A comparative study between cadaver, simulator, and training on live patients to evaluate speed of learning, frequency of complications to determine skills, and safety of performing endoscopic procedures can identify the advantages of various study models.

\section{Conclusion}

The use of human cadaver to train therapeutic endoscopic skills with predefined learning objectives can be performed on a teaching module.

\section{Authors' Contribution}

Avinash Bhat Balekuduru was involved in the concept of study, drafting of article, revising it, and final approval. Ashwini C. Appaji was involved in providing material of the study and concept of the study. 


\section{Conflict of Interest}

The authors declare that there is no conflict of interests regarding the publication of this paper. No grants were received for the study.

\section{Acknowledgments}

The authors would like to thank Dr Amit Kumar Dutta from CMC Vellore, Olympus Medical Systems, India, and Cook Medical for providing the accessories, Mr Ranjan B.S/Mr Nagendra from Ramaiah Medical College and all the postgraduate students/technical staff for their excellent assistance in the paper.

\section{References}

1 Balekuduru AB, Dutta AK, Subbaraj SB. Endoscopy on a human cadaver: a feasibility study as a training tool. J Digest Endosc 2018;09(3):103-108

2 Woods KL. Simulators in training for upper gastrointestinal endoscopy. Gastroenterol Hepatol (N Y) 2019;15(4):207-209

3 Khan R, Plahouras J, Johnston BC, Scaffidi MA, Grover SC, Walsh CM. Virtual reality simulation training for health professions trainees in gastrointestinal endoscopy. Cochrane Database Syst Rev 2018;8:CD008237, [PMID: 30117156 DOI: 10.1002/ 14651858.CD008237.pub3]

4 Khan R, Scaffidi MA, Grover SC, Gimpaya N, Walsh CM. Simulation in endoscopy: practical educational strategies to improve learning. World J Gastrointest Endosc 2019;11(3): 209-218

5 Issenberg SB, McGaghie WC, Petrusa ER, Lee Gordon D, Scalese RJ. Features and uses of high-fidelity medical simulations that lead to effective learning: a BEME systematic review. Med Teach 2005;27(1):10-28 [PMID: 16147767 DOI:10.1080/01421590500046924]
6 Hamstra SJ, Brydges R, Hatala R, Zendejas B, Cook DA Reconsidering fidelity in simulation-based training. Acad Med 2014;89(3):387-392 [PMID: 24448038 DOI: 10.1097/ ACM.0000000000000130]

7 Walsh CM, Cohen J, Woods KL, et al. ASGE EndoVators Summit: simulators and the future of endoscopic training. Gastrointest Endosc 2019;90(1):13-26

8 Siau K, Dunckley P, Valori R, et al; Joint Advisory Group on Gastrointestinal Endoscopy (JAG). Changes in scoring of Direct Observation of Procedural Skills (DOPS) forms and the impact on competence assessment. Endoscopy 2018;50(8):770-778

9 Walsh K. The rules. BMJ 2005;331(7516):574

10 Moulaert V, Verwijnen MG, Rikers R, Scherpbier AJJ. The effects of deliberate practice in undergraduate medical education. Med Educ 2004;38(10):1044-1052

11 Gauderer MWL, Ponsky JL, Izant RJ Jr. Gastrostomy without laparotomy: a percutaneous endoscopic technique. J Pediatr Surg 1980;15(6):872-875

12 Matthes K, Dunkin BJ, Jensen DM. Simulator training in endoscopic hemostasis. Tech Gastrointest Endosc 2011;13(3):174182 https://doi.org/10.1016/j.tgie.2011.07.001

13 Hochberger J, Matthes K, Maiss J, Koebnick C, Hahn EG, Cohen J. Training with the compactEASIE biologic endoscopy simulator significantly improves hemostatic technical skill of gastroenterology fellows: a randomized controlled comparison with clinical endoscopy training alone. Gastrointest Endosc 2005; 61(2):204-215

14 Matthes K, Cohen J, Kochman ML, Cerulli MA, Vora KC, Hochberger J. Efficacy and costs of a one-day hands-on EASIE endoscopy simulator train-the-trainer workshop. Gastrointest Endosc 2005;62(6):921-927

15 Blackburn SC, Griffin SJ. Role of simulation in training the next generation of endoscopists. World J Gastrointest Endosc 2014; 6(6):234-239 